\title{
Upregulating miR-27a-3p inhibits cell proliferation and inflammation of rheumatoid arthritis synovial fibroblasts through targeting toll-like receptor 5
}

\author{
LIFENG CHEN $^{1}$, QIPING LU ${ }^{2}$, JIANHUA CHEN ${ }^{1}$, RUIBING FENG $^{3}$ and CHENXI YANG ${ }^{4}$ \\ Departments of ${ }^{1}$ Rheumatology and Immunology and ${ }^{2}$ General Surgery, General Hospital of Central Theater Command; \\ ${ }^{3}$ Department of Orthopedics, Central People's Liberation Army Central Theater, \\ Wuhan, Hubei 430070; ${ }^{4}$ Department of Orthopedics, Graduate School of Hubei University of \\ Traditional Chinese Medicine, Wuhan, Hubei 430061, P.R. China
}

Received December 10, 2019; Accepted March 11, 2021

DOI: $10.3892 /$ etm.2021.10661

\begin{abstract}
Rheumatoid arthritis (RA) is a serious chronic inflammatory disease and synovial fibroblasts (SFs) serve a vital role in the pathogenesis and progression of RA. Current studies have demonstrated that dysregulation of microRNAs is involved in RA etiopathogenesis. The present study aimed to investigate the role of microRNA (miR)-27a-3p in RASFs, as well as its molecular mechanism. RASFs were isolated from synovial tissues from patients with RA. Expression of miR-27a-3p and toll-like receptor 5 (TLR5) was detected using reverse transcription-quantitative polymerase chain reaction and western blotting. Cell proliferation, apoptosis and inflammatory response were measured with MTT assay, flow cytometry and ELISA kits, respectively. The target binding between miR-27a-3p and TLR5 was predicted on DIANA TOOLS software, and confirmed by dual-luciferase reporter assay and Biotin-coupled miRNA pull-down assay. Expression of miR-27a-3p was downregulated and TLR5 was upregulated in synovial tissues and RASFs isolated from patients with RA. Functionally, upregulating miR-27a-3p may promote the apoptosis rate of RASFs and suppress cell proliferation and secretions of interleukin (IL)-1 $\beta$, IL-6 and tumor necrosis factor- $\alpha$. TLR5 was validated as a downstream target for miR-27a-3p in RASFs, and its expression was negatively regulated by miR-27a-3p. Silencing TLR5 in RASFs may exert similar effects to miR-27a-3p-overexpression; whereas, restoring TLR5 counteracted the suppression of miR-27a-3p-overexpression on RASF proliferation and inflammation, as well as the promotion on apoptosis. miR-27a-3p
\end{abstract}

Correspondence to: Mr. Lifeng Chen, Department of Rheumatology and Immunology, General Hospital of Central Theater Command, 627 Wuyi Road, Wuchang, Wuhan, Hubei 430070, P.R. China E-mail: ghbjof@163.com

Key words: miR-27a-3p, TLR5, rheumatoid arthritis synovial fibroblasts upregulation may suppress RA progression by inhibiting RASFs proliferation and inflammation through targeting TLR5.

\section{Introduction}

Rheumatoid arthritis (RA) is a debilitating, systemic and chronic autoimmune disease (1). The characteristics of RA include synovial hyperplasia, joint destruction and extra-articular manifestations, which finally leads to tissue destruction (2). A combination of gene, environment and immunology factors has been well-documented to be responsible for the onset and development of RA (3). Furthermore, multiple inflammatory mediators (small factors that are secreted by cells and then induce an inflammatory response), immune cells and non-immune cells are collectively involved in the inflammatory processes in RA (4). Interleukin (IL)-1 $\beta$, IL-6, IL-17, IL-22, IL-23 and tumor necrosis factor (TNF)- $\alpha$ are inflammatory mediators that serve an important role in RA mainly at the synovial level, evoking significant pathological changes (5); among these changes, the infiltration of self-reactive $\mathrm{T}$ and B cells, including Th17 cells (immune cells), initiates a complex autoimmune response and produces certain cytokines and proliferation factors that drive fibroblasts (non-immune cells) differentiate into synoviocyte-like cells, called synovial fibroblasts (SFs) (6). Furthermore, loss of balance in SF proliferation and apoptosis leads to hypertrophied synovium or pannus formation, which is a hallmark of RA (7).

At present, clinically used drugs for RA have been advanced, including nonsteroidal anti-inflammatory drugs, immunosuppressive agents, glucocorticoids and biological agents (8). However, only 50-60\% of patients with RA respond to these drugs owning to their inherent limitations in the clinical treatment of RA (9). Over the past few years, certain studies have suggested that genetic approach may offer novel therapeutic methods for RA $(6,10)$. Furthermore, microRNAs (miRNAs) have been revealed to demonstrate diverse crucial roles in the pathological process of RA (11). miRNAs are short, non-coding RNA sequences that repress gene expression 
generally by binding to the 3'-untranslated region (3'-UTR) of target messenger RNAs (mRNAs). Accumulating data have revealed miRNAs as multifunctional regulators in numerous physiological processes, including proliferation, apoptosis and differentiation (12). Pathologically, miRNAs have been extensively studied and applied as biomarkers and targets for cancers. Furthermore, recent studies have indicated that miRNAs are gaining increasing recognition for their involvement in autoimmune diseases (13-15), including RA. Altered expression of miRNAs is reported to be associated with the occurrence and development of RA $(16,17)$.

Several studies have evaluated miRNAs expression in RA patient samples, including plasma, synovial tissue and SFs (18-20). Among these miRNAs, miR-27a-3p is downregulated in patients with $\mathrm{RA}$ and serves a crucial role in RASF migration and invasion, which are partly answerable for the spread of arthritic destruction to distant joints $(18,21)$. Furthermore, expression of miR-27a-3p is associated with the patient's responsiveness to therapy $(13,18)$. However, its role in RA has not been completely elucidated. Based on the existing data stating the dysregulation of miR-27a-3p in synovial tissues and SFs from patients with RA $(13,18,21)$, and the present bioinformatics data from DianaTools (http://diana. imis.athena-innovation.gr/DianaTools/index.php?r=site/index) predicting the association between miR-27a-3p and TLRs; therefore, $\mathrm{miR}-27 \mathrm{a}-3 \mathrm{p}$ and TLR5 received attention to be further investigated regarding their role in RA pathogenesis and progression in RASFs.

The present study isolated human synovial tissues and RASFs from patients with RA and further investigated the expression of miR-27a-3p. The functional roles of miR-27a-3p and TLR5 in RASF proliferation, apoptosis and inflammation were determined, as well as the association between miR-27a-3p and TLR5.

\section{Materials and methods}

Collection of tissue samples. The present study was performed according to the recommendations of the Declaration of Helsinki. A total of 27 patients with RA and 27 non-RA control subjects between January 2013 and December 2016 were recruited from General Hospital of Central Theater Command (Wuhan, China). The present study was performed with the approval of the Research Ethics Committee of General Hospital of Central Theater Command. The patients with RA were diagnosed with the 2010 American College of Rheumatology/European League against Rheumatism classification criteria (2010 ACR/EULAR) for RA (22). The control subjects were patients with osteoarthritis and joint trauma but free of autoimmune disease and infectious disease. Patients with RA who achieved a score of at least 6 out of 10 points according to the $2010 \mathrm{ACR} / \mathrm{EULAR}$ were included in the present study. The exclusion criteria for the patients were as follows: i) Presence of malignant diseases such as cancer; ii) presence of central or peripheral nervous system diseases such as trigeminal neuralgia; iii) presence of cardiac diseases such as coronary heart disease, and iv) presence of endocrine disorders (especially diabetes mellitus). The demographic characteristic of patients with RA are presented in Table I. The synovial tissue samples from patients were obtained while undergoing joint surgery at the hospital. Tissue samples were obtained following the written informed consents being obtained from all patients. All tissue samples were immediately frozen in liquid nitrogen and then stored at $-80^{\circ} \mathrm{C}$ prior to use.

Isolation and culture of RASFs. As previously described (23), RASFs and control synovial fibroblasts (CSFs) were isolated from eight indicated patients, respectively. In short, synovial tissues were washed with sterile phosphate buffer solution (PBS), followed by being minced and digested using $1 \mathrm{mg} / \mathrm{ml}$ collagenase type II (Invitrogen; Thermo Fisher Scientific, Inc.) for $4 \mathrm{~h}$ at $37^{\circ} \mathrm{C}$. The cells were collected and incubated in RPMI-1640 (HyClone; GE Healthcare Life Sciences), containing $10 \%$ fetal bovine serum (HyClone; GE Healthcare Life Sciences) and $4 \mathrm{mM}$ glutamine at $37^{\circ} \mathrm{C}$ in $5 \% \mathrm{CO}_{2}$ for 1 month with a change of culture medium every 3 days. All experiments were conducted using cultured cells between passages 3-6.

Cell transfection. RASFs were seeded into 6-well plates (Corning Incorporate) for $24 \mathrm{~h}$ prior to transfection. Plasmids $(3 \mu \mathrm{g})$ and miRNA/siRNA $(100 \mathrm{nM})$ were transfected into RASFs using Lipofectamine ${ }^{\circledR} 3000$ (Invitrogen; Thermo Fisher Scientific, Inc.) at $37^{\circ} \mathrm{C}$ for $4-6 \mathrm{~h}$, according to the manufacturer's protocol. Transfected miR-27a-3p mimic (miR-27a-3p; 5'-UUCACAGUGGCUAAGUUCCGC-3'), miR-27a-3 p inhibitor (in-miR-27a-3p; 5'-GCGGAACUUAGCCACUGUGAA-3') and siRNA targeting toll like receptor 5 (si-TLR5; 5'-CUGUGA UGAGAUUCCUAUAGU-3' and 5'UAUAGGAAUCUCAUC ACAGUG-3') were purchased from Guangzhou Ribobio Co., Ltd., as well as the negative controls miR-NC (5'-ACGUGACAC GUUCGGAGAATT-3'), in-miR-NC (UUCUCCGAACGUGUC ACGUTT) and si-NC ( 5 ' U UCUCCGA A C GUGUCACGUT T-3' and 5'ACGUGACACGUUCGGAGAATT-3'). The full-length human TLR5 coding domain sequence was cloned into pcDNA4.1 (Invitrogen; Thermo Fisher Scientific, Inc.). In rescue experiments, co-transfection was launched with $1.5 \mu \mathrm{g}$ plasmid and $60 \mathrm{nM}$ miR-27a-3p or miR-NC. Cells were subsequently cultured for another $30 \mathrm{~h}$ prior to further study.

RNA extraction and reverse transcription-quantitative polymerase chain reaction ( $R T-q P C R)$. Total RNA was extracted from tissue samples and RASFs using Qiagen miRNeasy Mini kit (Qiagen $\mathrm{GmbH}$ ), according to the manufacturer's protocol. The cDNAs were synthesized depending on total RNA and a reverse transcription kit (Abcam, Canada). The reverse transcription protocol was as follows: $25^{\circ} \mathrm{C}$ for $5 \mathrm{~min}$, $55^{\circ} \mathrm{C}$ for $15 \mathrm{~min}$ and $85^{\circ} \mathrm{C}$ for $5 \mathrm{~min}$. The amplification of cDNAs was performed using a SYBR ${ }^{\circledR}$-Green Master mix kit (Qiagen $\mathrm{GmbH}$ ) on an Applied Biosystems 7500 Real-Time PCR system (Thermo Fisher Scientific, Inc.). The primers were as follows: miR-27a-3p forward, 5'-ACACTCCAGCTGGGTTCACAGTG GCTAAG-3' and reverse, 5'-AGGGCTTAGCTGCTTGTGA GCA-3'; TLR5 forward, 5'-TGCCACTGTTGAGTGCAAGTC-3' and reverse, 5'-ACCTGGAGAAGCCGAAGGTAA-3'; GAPDH forward, 5'-GACAGTCAGCCGCATCTTCT-3' and reverse, 
Table I. Demographic characteristics and miR-27a-3p expression in synovial tissue samples from patients with RA.

\begin{tabular}{lccr}
\hline Features & Low miR-27a-3p expression $(\mathrm{n}=15)$ & High miR-27a-3p expression $(\mathrm{n}=12)$ & P-value \\
\hline Age, mean \pm SD & $52.43 \pm 8.59$ & $51.35 \pm 10.25$ & 0.698 \\
Male/female, $\mathrm{n}$ & $7 / 8$ & $5 / 7$ & 0.325 \\
CRP, mg/l & $35.57 \pm 15.33$ & $20.35 \pm 10.25$ & $0.037^{\mathrm{a}}$ \\
DAS28 & $13.59 \pm 3.58$ & $5.21 \pm 1.02$ & $0.003^{\mathrm{a}}$ \\
ESR, mm/h & $22.33 \pm 3.83$ & $14.08 \pm 2.65$ & $0.046^{\mathrm{a}}$ \\
RF positive, $\%$ & 56.25 & 51.39 & 1.029 \\
Anti-CCP & $198.25 \pm 56.32$ & $193.57 \pm 42.85$ & 1.058 \\
Swollen joint count & $2.56 \pm 0.58$ & $0.53 \pm 0.22$ & $0.024^{\mathrm{a}}$ \\
\hline
\end{tabular}

miR, microRNA; RA, rheumatoid arthritis; SD, standard deviation; CRP, C-reactive protein; DAS28, disease activity score of 28-joint; ESR, erythrocyte sedimentation rate; RF, rheumatoid factor; Anti-CCP: anti-cyclic citrullinated peptide. ${ }^{\text {aP }}<0.05$.

5'-GCGCCCA ATACGACCAAA-3'; U6 forward, 5'-GCTTCGGCAGCACATATACTAAAAT-3' and reverse, 5'-CGCTTCACGAATTTGCGTGTCAT-3'. The thermocycling conditions used were $95^{\circ} \mathrm{C}$ for $10 \mathrm{~min}$, followed by 40 cycles of $95^{\circ} \mathrm{C}$ for $15 \mathrm{sec}, 60^{\circ} \mathrm{C}$ for $30 \mathrm{sec}$ and $60^{\circ} \mathrm{C}$ for $15 \mathrm{sec}$. The reaction was held at $74^{\circ} \mathrm{C}$ for continuous for melting curve analysis. The relative expression levels of miR-27a-3p and TLR5 mRNA were calculated by $2^{-\Delta \Delta C T}$ methods (24) with normalization to U6 small nuclear RNA (U6) and GAPDH, respectively. All PCR reactions were performed in triplicate.

Protein extraction and western blotting. Total protein was extracted from cultivated RASFs using RIPA lysis buffer (Beyotime Institute of Biotechnology), and protein concentration was determined using the BCA method. TLR5 protein expression was measured using western blotting, which was performed according to standard procedures (25). A total of $20 \mu \mathrm{g}$ was protein per lane lane and separated using $8-10 \%$ SDS-PAGE. PVDF membranes (EMD Millipore) were blocked with 3\% BSA (Sigma-Aldrich; Merck KGaA) at $25^{\circ} \mathrm{C}$ for $1 \mathrm{~h}$. $\beta$-actin on the same membrane was used as the loading control. The antibodies were purchased from Abcam and were as follows: anti-TLR5 (1:2,000; cat. no. ab168382), anti- $\beta$-actin (1:10,000; cat. no. ab227387;), horse-radish peroxidase (HRP)-labelled anti-Rabbit IgG (1:50,000; cat. no. ab205718). Primary antibody incubation was at $4^{\circ} \mathrm{C}$ overnight and secondary antibody incubation was performed at $25^{\circ} \mathrm{C}$ for $1 \mathrm{~h}$. The proteins were visualized using ECL procedure (EMD Millipore), and ImageJ v1.8.0 software (National Institutes of Health, Bethesda) was used to analyze the gray intensity of the bands.

MTT assay. Transfected RASFs (5,000 cells) were seeded onto 96-well plates (Corning Incorporated) for 0, 24, 48 and $72 \mathrm{~h}$. The cell proliferation was determined by MTT staining. $5 \mathrm{mg} / \mathrm{ml}$ MTT (20 $\mu \mathrm{l}$; Sigma-Aldrich; Merck KGaA) was added for another $4 \mathrm{~h}$-incubation; the formazan was dissolved in $150 \mu$ l dimethyl sulfoxide (Sigma-Aldrich; Merck KGaA). The absorbance at $450 \mathrm{~nm}$ was measured on a microplate reader (Molecular devices, LLC). All experiments were performed in triplicate.
Apoptosis assay. The apoptotic rate of transfected RASFs was analyzed using a Annexin V-FITC/propidium iodide (PI) kit (Beyotime Institute of Biotechnology) using flow cytometry. Following transfection for $30 \mathrm{~h}$, apoptotic cells were dual-labelled with Annexin V-FITC and PI for $30 \mathrm{~min}$ in the dark, and fluorescence was analyzed on a cytoFLEX LX flow cytometer (Beckman Coulter, Inc.), supplemented with CytExpert v.2.0 software (Beckman Coulter, Inc.). Quadrants were positioned on Annexin V/PI plots to distinguish apoptotic cells (Annexin $\mathrm{V}^{+} / \mathrm{PI}^{-}$, Annexin $\mathrm{V}^{+} / \mathrm{PI}^{+}$). Apoptosis $(\%)=$ apoptotic cells/total cells $\times 100 \%$.

ELISA. ELISA was conducted to measure the concentrations of IL-1 $\beta$, IL- 6 and TNF- $\alpha$ released in the culture medium of RASFs. Following transfection for $30 \mathrm{~h}$, the culture supernatant of RASFs was collected and subjected to the ELISA kits purchased from Abcam: Human IL-1 $\beta$ ELISA kit (cat. no. ab100562), human IL-6 ELISA kit (cat. no. ab46027) and human TNF- $\alpha$ ELISA kit (cat. no. ab46087). All operations were performed according to the manufacturer's protocols, and the reactions were set in triplicate for each sample.

Bioinformatics analysis. According to in silico data on DIANA TOOLS v3.0 (DianaTools; http://diana.imis. athena-innovation.gr/DianaTools/index.php?r=site/index), potential binding sites of TLR5 (ENSG00000187554) and hsa-miR-27a-3p were investigated.

Luciferase reporter assay. The fragment sequence of TLR5 3'-UTR containing the potential binding sites of miR-27a-3p was cloned by PCR methods into pGL4 vector (Invitrogen; Thermo Fisher Scientific, Inc.) to construct pGL4-TLR5 3'-UTR-WT vectors; similarly, pGL4-TLR5 3'-UTR-MUT vectors were constructed to carry the TLR5 3'-UTR fragment containing the mutations of miR-27a-3p responsive elements. RASFs were co-transfected with pGL4-TLR5 3'-UTR-WT/MUT vectors and miRNA mimics or inhibitors for $48 \mathrm{~h}$ using Lipofectamine ${ }^{\circledR} 3000$ reagent (Invitrogen; Thermo Fisher Scientific, Inc.), and cell lysate was collected to measure Firefly and Renilla luciferase activities using the dual-luciferase reporter assay system (Promega Corporation). At least three independent transfections were set in each 

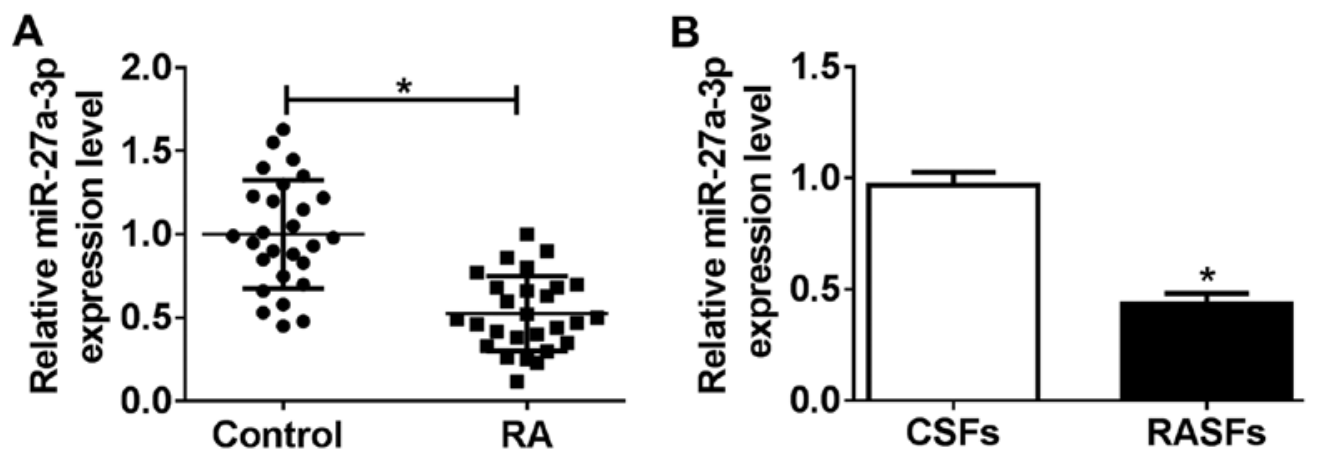

Figure 1. Expression of miR-27a-3p in synovial tissues from patients with RA and RASFs. Reverse transcription-quantitative polymerase chain reaction measured the miR-27a-3p level in (A) synovial tissues from patients with RA ( $n=27)$ and non-RA controls ( $n=27)$, and (B) RASFs and CSFs. * $<0.05$, compared with Control and CSFs. miR, microRNA; RA, rheumatoid arthritis; RASFs, RA synovial fibroblasts; CSFs, control synovial fibroblasts.

group and the relative luciferase activity was presented as fold-change normalized to the negative control group.

Biotin-coupled miRNA pull-down assay. Biotin-labelled miR-27a-3p mimic (Bio-miR-27a-3p; biotin-UUCACAGUGGCUAAGUUCC) and biotin-labelled miR-27a-3p-MUT mimic (Bio-miR-27a-3p MUT; bio-AAGUGUCUGGCUAAGUUCC), as well as biotin-labelled miR-NC mimic (Bio-miR-NC; biotin-UUCUCCGAACGUGUCACGU) were synthesized by Guangzhou Ribobio Co., Ltd. RASFs were transfected with $50 \mu \mathrm{M}$ of the aforementioned biotinylated miRNAs for $30 \mathrm{~h}$. Next, cell lysates of transfected RASFs were obtained by lysing cells in lysis buffer and sonicate. Subsequently, cell lysates were incubated with $50 \mu \mathrm{l}$ streptavidin-coated magnetic beads (Sigma-Aldrich; Merck KGaA) at room temperature for $2 \mathrm{~h}$, and biotin-coupled miRNA capture was subjected to Qiagen miRNeasy Mini kit (Qiagen $\mathrm{GmbH}$ ) to recycle the precipitated RNAs. Expression of TLR5 mRNA in the aforementioned precipitated RNAs was analyzed using RT-qPCR.

Statistical analysis. Statistics were analyzed by SPSS 21.0 (IBM Corp.) and data are presented as the mean \pm standard deviation (Table SI). The independent samples t-test method was utilized for comparison between two groups, and one-way analysis of variance (ANOVA) was for comparison among multiple groups. Tukey's post hoc test was applied following ANOVA. $\mathrm{P}<0.05$ was considered to indicate a statistically significant difference.

\section{Results}

Expression of miR-27a-3p is downregulated in human $R A$ synovial tissues and RASFs. To investigate the role of miR-27a-3p in pathological and physiological changes in patients with RA, the present study investigated miR-27a-3p expression in RA specimens. According to RT-qPCR analysis, miR-27a-3p levels were significantly downregulated (0.51-fold; $\mathrm{P}<0.05)$ in RA synovial tissues, compared with that from non-RA controls (Fig. 1A). Furthermore, RASFs and CSFs were isolated from eight patients with RA and eight non-RA controls, respectively. As shown in Fig. 1B, the level of miR-27a-3p was decreased $(0.45$-fold; $\mathrm{P}<0.05)$ in RASFs, compared with that in CSFs. Furthermore, low expression of
miR-27a-3p was correlated with several RA features, including a disease activity score of 28-joint (DAS28), swollen joint count, C-reactive protein (CRP) level and erythrocyte sedimentation rate (ESR; Table I). Therefore, we hypothesized that dysregulation of miR-27a-3p may serve a potential role in RASF dysfunction, and that miR-27a-3p may be a potential clinical diagnostic marker in RA.

Upregulation of miR-27a-3p suppresses proliferation and inflammatory response of human RASFs. Isolated RASFs were cultured and miR-27a-3p was highly expressed ex-vivo. Gain-of-function experiments were performed in RASFs following mimic transfection. To begin with, transfection efficiency was validated by RT-qPCR, and miR-27a-3p was significantly overexpressed $(3.7$-fold; $\mathrm{P}<0.05)$ in $\mathrm{RASFs}$ in the presence of exogenous miR-27a-3p, compared with miR-NC (Fig. 2A). The MTT assay demonstrated that the cell proliferation of RASFs was attenuated by miR-27a-3p mimic transfection (Fig. 2B). FCM revealed a distinctive augmentation of apoptosis rate (from 8.3 to $30.7 \%$; $\mathrm{P}<0.05$ ) in RASFs transfected with miR-27a-3p with normalization to control transfection (Fig. 2C). Furthermore, the secretions of inflammatory cytokines were detected by ELISA kits, and products of IL-6, IL-1 $\beta$ and TNF- $\alpha$ were markedly declined in RASFs with miR-27a-3p-overexpression via transfection (Fig. 2D-F). These results demonstrated that miR-27a-3p upregulation may promote apoptosis and suppress proliferation and inflammation of RASFs.

TLR5, serving as a downstream target for $m i R-27 a-3 p$, is upregulated in patients with $R A$. Bioinformatics analysis indicated that TLR5 was a potential one (Fig. 3A). There were potential responsive elements of miR-27a-3p 'seed sequence' (UCACAG) in TLR5 3'-UTR: position 157-169 (conserved). To confirm this prediction, dual-luciferase reporter and biotin-coupled miRNA pull-down assays were conducted. The relative luciferase activity of pGL4-TLR5 3'-UTR-WT vectors was significantly decreased $(0.35$-fold; $\mathrm{P}<0.05)$ in RASFs with miR-27a-3p-overexpression via mimic transfection, and was increased $(2.50$-fold; $\mathrm{P}<0.05)$ with miR-27a-3p-silencing via inhibitor transfection (Fig. 3B and C). However, there was no difference in pGL4-TLR5 3'-UTR-MUT vector-transfected RASFs when miR-27a-3p was overexpressed or downregulated. Furthermore, TLR5 expression was 
A

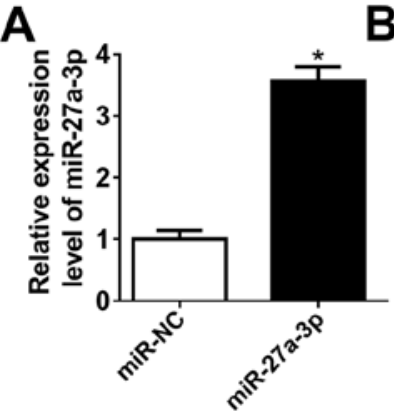

D

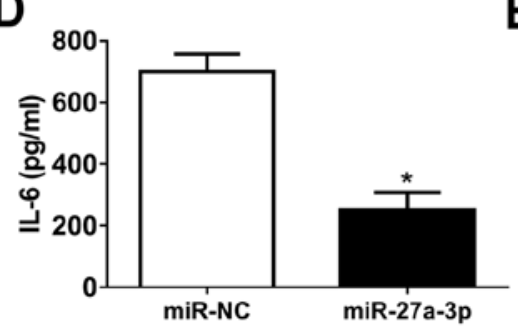

B

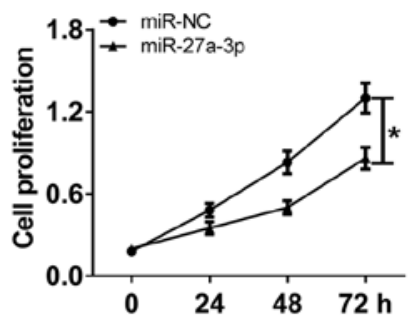

E

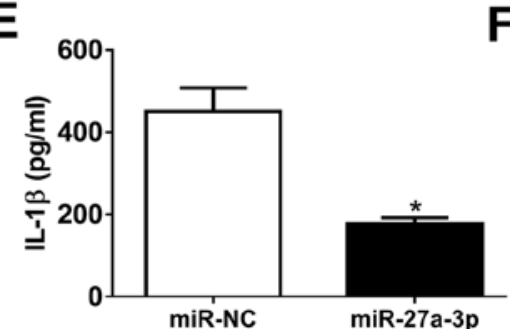

C
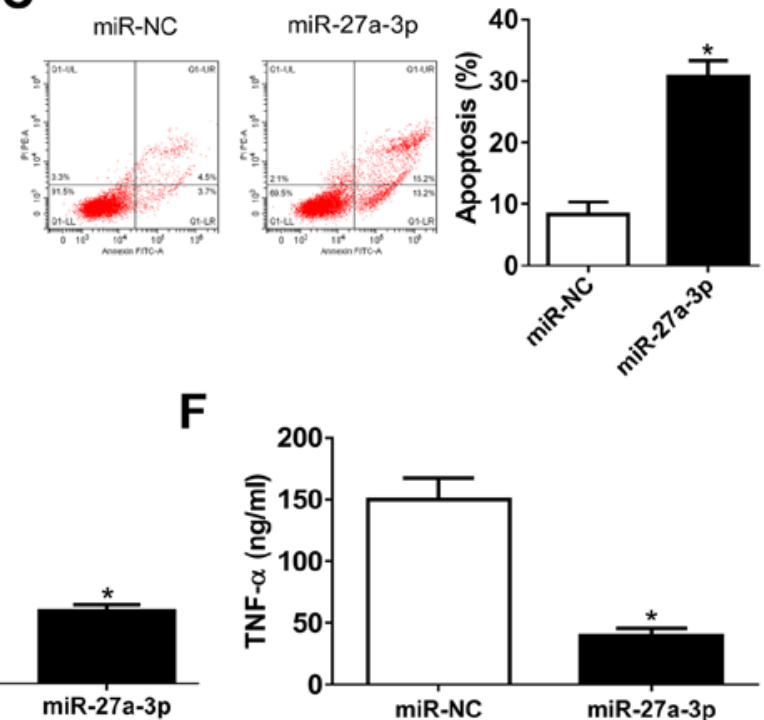

Figure 2. Role of miR-27a-3p in the proliferation, apoptosis and inflammation of RASFs. miR-27a-3p was exogenously overexpressed in RASFs by transfecting miR-27a-3p mimic (miR-27a-3p). (A) Reverse transcription-quantitative polymerase chain reaction assay detected the miR-27a-3p level following transfection. (B and C) Cell proliferation and apoptosis were investigated using MTT assay and flow cytometry. (D-F) Products of IL-6, IL-1 $\beta$ and TNF- $\alpha$ were determined by ELISA kits. ${ }^{*}<0.05$, compared with miR-NC mimic (miR-NC). miR, microRNA; RASFs, RA synovial fibroblasts; IL, interleukin; TNF, tumor necrosis factor; NC, negative control.

significantly enriched (4.43-fold; $\mathrm{P}<0.05)$ by Bio-miR-27a-3p instead of Bio-miR-27a-3p-MUT, compared with the control group (Fig. 3D). Additionally, the expression of TLR5 in RA was also investigated. As shown in Fig. 3E and F, expression levels of TLR5 were significantly upregulated (2.14-fold and 2.27-fold; $\mathrm{P}<0.05)$ in synovial tissues and RASFs from patients with RA. Western blotting revealed that TLR5 protein expression was decreased (0.32-fold; $\mathrm{P}<0.05)$ in miR-27a-3p-overexpressed RASFs via mimic transfection, and was increased (2.20-fold; $\mathrm{P}<0.05$ ) in miR-27a-3p-silenced RASFs (Fig. 3G). These data suggested that TLR5 was a downstream target for miR-27a-3p, and its expression was upregulated in patients with RA.

Silencing of TLR5 depresses proliferation and inflammatory response in human RASFs. Loss-of-function experiments were performed in RASFs transfected with si-TLR5 or si-NC. The transfection efficiency was first validated by western blotting, and TLR5 protein expression was distinctively downregulated $(0.31$-fold; $\mathrm{P}<0.05)$ in the presence of si-TLR5, compared with si-NC (Fig. 4A). Cell proliferation was inhibited and the apoptosis rate was promoted in RASFs with si-TLR5 transfection, as evidenced by MTT assay and FCM method (Fig. 4B and C). Furthermore, ELISA kits revealed that the secretions of IL-6, IL-1 $\beta$ and TNF- $\alpha$ were markedly decreased in RASFs with TLR5-knockdown via transfection (Fig. 4D-F). These results demonstrated that TLR5 downregulation may promote apoptosis and suppress proliferation and inflammation in RASFs, which was similar to role of miR-27a-3p upregulation (Fig. 2A-F).

Restoration of TLR5 counteracts the suppressive effect of miR-27a-3p-overexpression on human RASF proliferation and inflammatory response. The present study aimed to investigate whether miR-27a-3p exerted a suppressive effect on RASF proliferation and inflammation through inhibiting its target TLR5. Therefore, rescue experiments were performed. RASFs were co-transfected with miR-27a-3p and a pcDNA-TLR5 (TLR5) vector or pcDNA vector. Expression of TLR5 was measured by western blotting following transfection; the decreased TLR5 protein levels mediated by miR-27a-3p overexpression were improved (from 0.37-fold to 0.83 -fold; $\mathrm{P}<0.05$ ) in the co-transfection of miR-27a-3p mimic and TLR5 vector (Fig. 5A). Cell proliferation of RASFs was inhibited by miR-27a-3p-overexpression, and then was rescued when miR-27a-3p and TLR5 were concurrently overexpressed (Fig. 5B). miR-27a-3p mimic-induced a higher apoptosis rate in RASFs, which was decreased (from 28.0 to $15.6 \%$; $\mathrm{P}<0.05$ ) by also transfecting the TLR5 vector (Fig. 5C). Furthermore, TLR5 upregulation via vector transfection reversed the inhibitory effect of miR-27a-3p mimic on the secretions of IL- 6 , IL- $1 \beta$ and TNF- $\alpha$ in RASFs (Fig. 5D-F). These results demonstrated that restoration of TLR 5 counteracted the biological effects of miR-27a-3p-overexpression in RASFs, suggesting a miR-27a-3p/TLR5 axis in proliferation, apoptosis and inflammation of RASFs (Fig. 6).

\section{Discussion}

The prevalence of RA varied from $0.3-1.0 \%$ globally, and it was more common in developed countries than others (26). Although current therapies offer diverse choices for RA drugs, only 50-60\% of patients with RA responded to them (9). Aberrant expression of miRNAs was associated with the patients' responsiveness to therapy in RA. For example, Dudics et al (13) observed that 8 special miRNAs had the potential to be key regulators of arthritis pathogenesis; among these, 6 miRNAs may serve as biomarkers of therapeutic response of celastrol in patients with RA. Furthermore, miR-27a-3p was one of the 8 upregulated miRNAs following RA development, but was downregulated in response to celastrol treatment. In a placebo-controlled clinical trial, Sode et al (18) confirmed that the high miR-27a-3p pretreatment level and the decrease in the miR-27a-3p level 


\section{A \\ hsa-miR-27a-3p TLR5 3'UTR-WT TLR5 3'UTR-MUT}

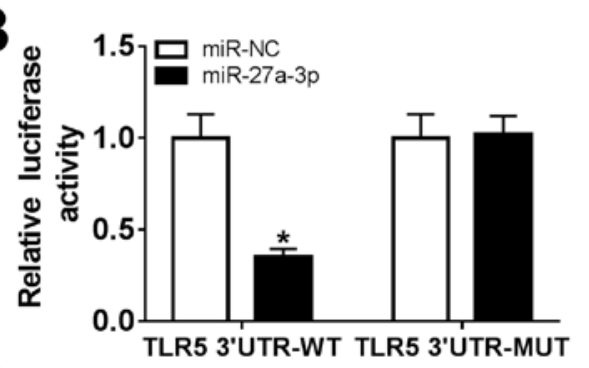

D

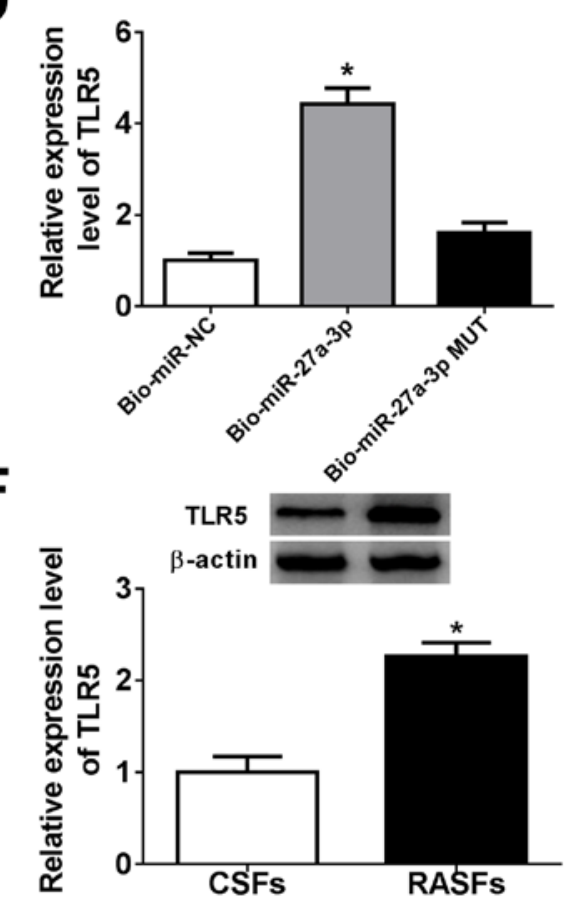

3' CCUUGAAUCGGUGACACUU 5' 5' ...GUCAUAGAAUUCCUGUGAA... 3' 5' ...GUCAUAGAAUUCGACACUU... 3'

C

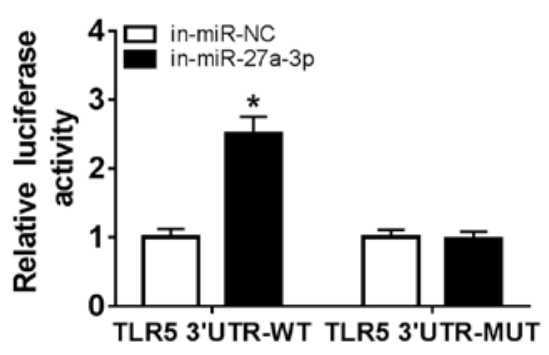

E

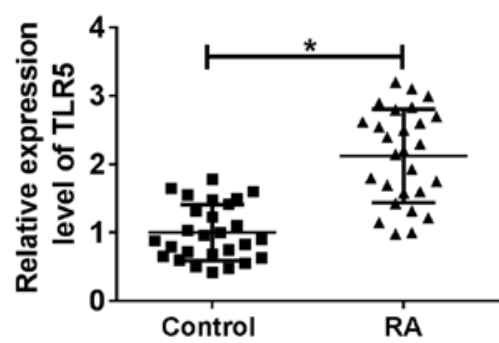

G

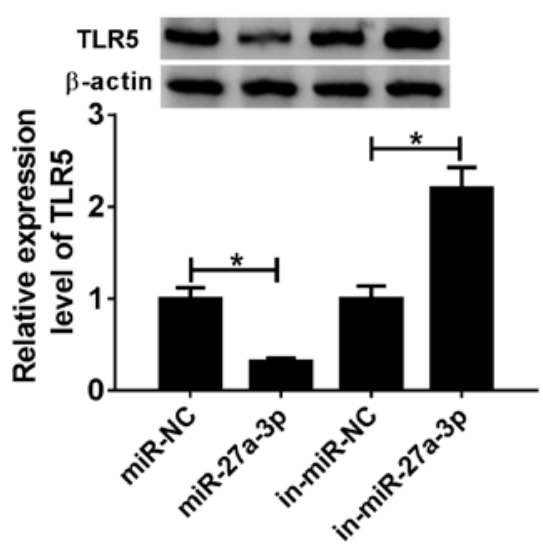

Figure 3. miR-27a-3p negatively regulates TLR5 expression by target binding. (A) Schematic diagram demonstrating the potential miR-27a-3p responsive elements in wild-type and mutant of TLR5 3'-UTR (TLR5 3'-UTR-WT and TLR5 3'-UTR-MUT). (B and C) Dual-luciferase reporter assay detected the luciferase of pGL4-TLR5 3'-UTR-WT/MUT vectors in RASFs co-transfected with miR-27a-3p/NC or miR-27a-3p/NC inhibitors (in-miR-27a-3p/NC). ${ }^{*} \mathrm{P}<0.05$, compared with the miR-NC group or in-miR-NC group. (D) RNA pull-down assay measured the TLR5 level in RASFs transfected with biotin-labelled miR-27a-3p (Bio-miR-27a-3p) or its mutant (Bio-miR-27a-3p MUT). ${ }^{*} \mathrm{P}<0.05$, compared with biotin-labelled miR-NC (Bio-miR-NC). (E) Reverse transcription-quantitative polymerase chain reaction detected the TLR5 mRNA level in RA synovial tissue $(\mathrm{n}=27)$ and Control synovial tissue ( $\mathrm{n}=27)$. ${ }^{*} \mathrm{P}<0.05$, compared with the Control. (F) Western blotting investigated the TLR5 protein level in RASFs and CSFs. * $<<0.05$, compared with CSFs. (G) Western blotting investigated the TLR5 protein level in RASFs transfected with miR-27a-3p/NC or in-miR-27a-3p/NC. * $\mathrm{P}<0.05$, compared with miR-NC or in-miR-NC. miR, microRNA; TLR5, toll-like receptor 5; UTR, untranslated region; WT, wild-type; MUT, mutant; RASFs, RA synovial fibroblasts; NC, negative control; in, inhibitor; RA, rheumatoid arthritis; CSFs, control synovial fibroblasts.

with treatment for 3 months was associated with ACR/EULAR Boolean remission at 12 months. Furthermore, miR-16-5p and miR-22-3p pretreatment levels and their level changes over 3-months treatment served as predictive target of MTX response after 3 and 12 months, respectively. These results proposed that certain miRNAs, including miR-27a-3p, may serve as potent biomarkers to monitor disease activity and therapeutic response in RA.

By contrast to the other fibroblasts, RASFs had a high-level of invasive ability (25), and shared certain similar features with cancer cells (27), including migration, invasion and resistance to apoptosis. Synoviocyte migration may be essential to the pathology of RA, and contributed toward the spread of arthritic destruction to distant joints (28). On one hand, abnormal miRNA expression may cause cell migration abnormality and inflammation in RASFs. For instance, miR-27a-5p downregulation underlies the molecular mechanism of lncRNA ZFAS1 promoting human RASF migration and invasion (29). By contrast, downregulation of miR-221 decreased RASF migration and invasion via inhibiting expression of vascular 


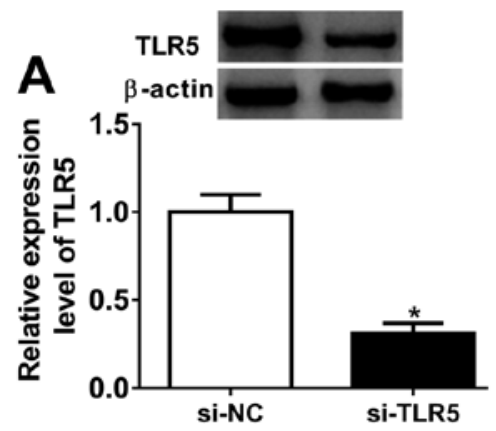

B

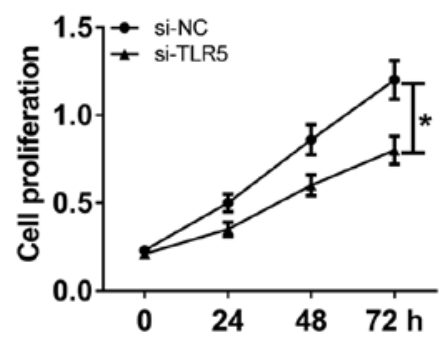

C
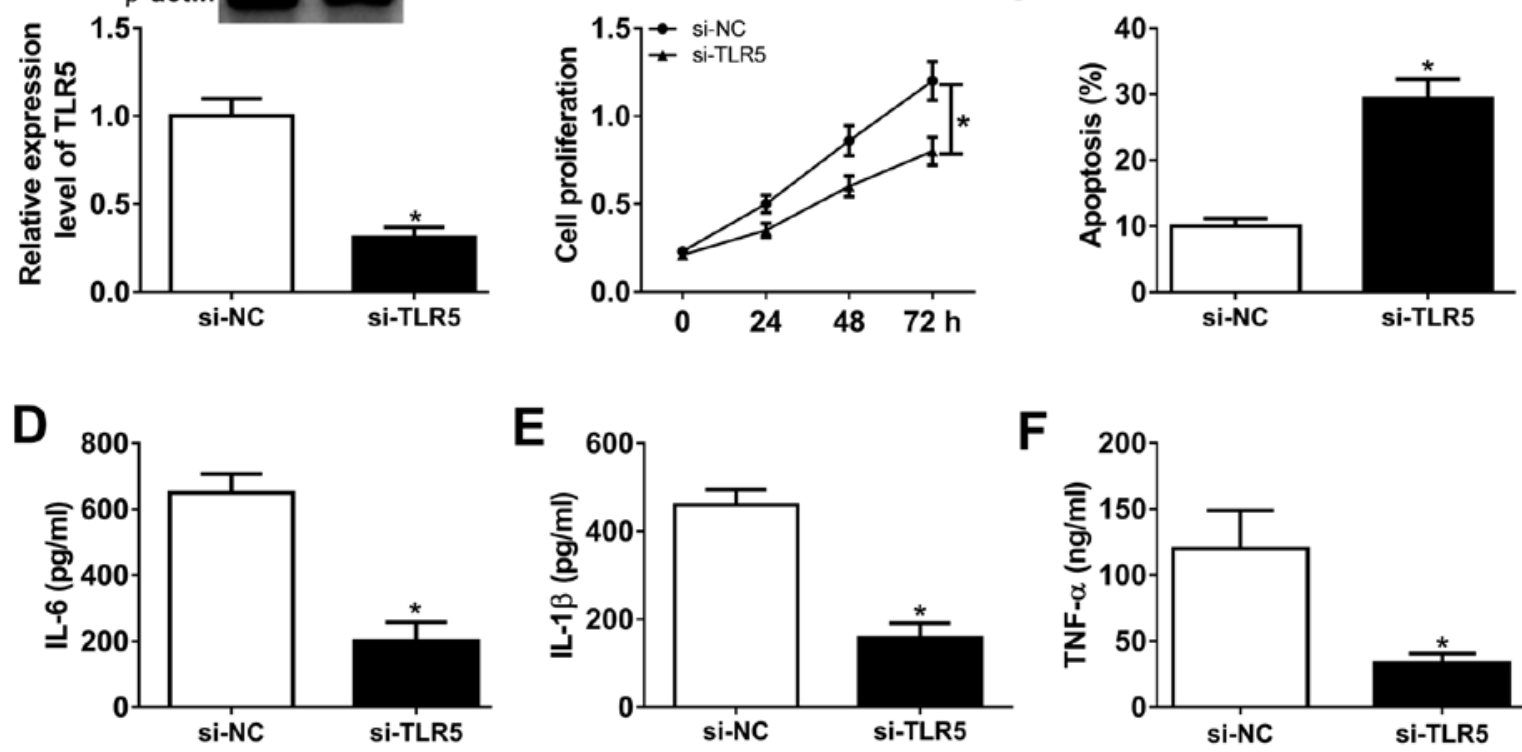

$\mathbf{E}$

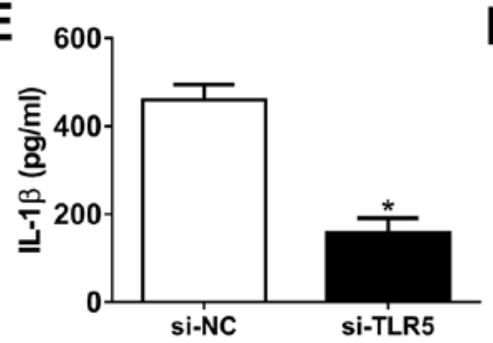

$\mathbf{F}$

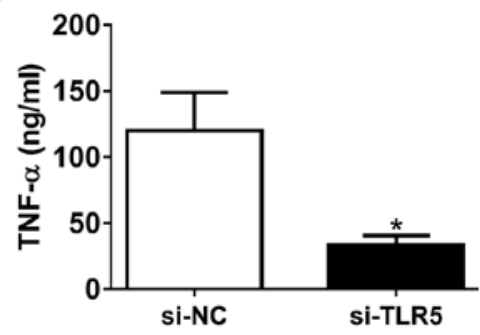

Figure 4. Effect of TLR5 on the proliferation, apoptosis and inflammation in RASFs. TLR5 was exogenously silenced in RASFs by transfecting siRNA targeting TLR5 (si-TLR5). (A) Western blotting detected the TLR5 level. (B and C) Cell proliferation and apoptosis were investigated using MTT assay and flow cytometry. (D-F) Products of IL-6, IL- $1 \beta$ and TNF- $\alpha$ were determined by ELISA kits. ${ }^{*} \mathrm{P}<0.05$, compared with scrambled siRNA (si-NC). TLR5, toll-like receptor 5; RASFs, RA synovial fibroblasts; NC, negative control; IL, interleukin; TNF, tumor necrosis factor. TLR5, toll-like receptor 5; RASFs, RA synovial fibroblasts; IL, interleukin; TNF, tumor necrosis factor; NC, negative control.
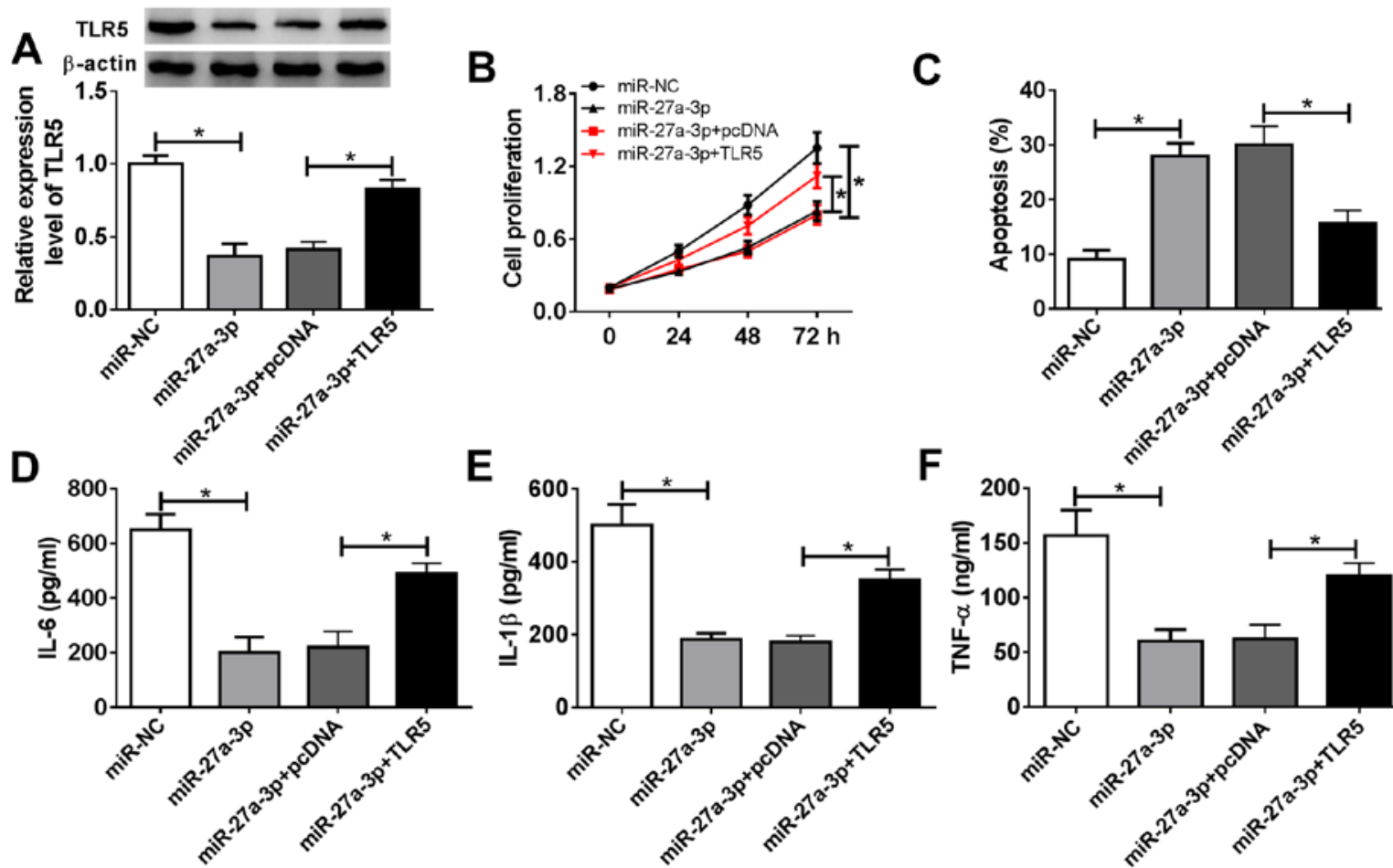

$\mathbf{E}$
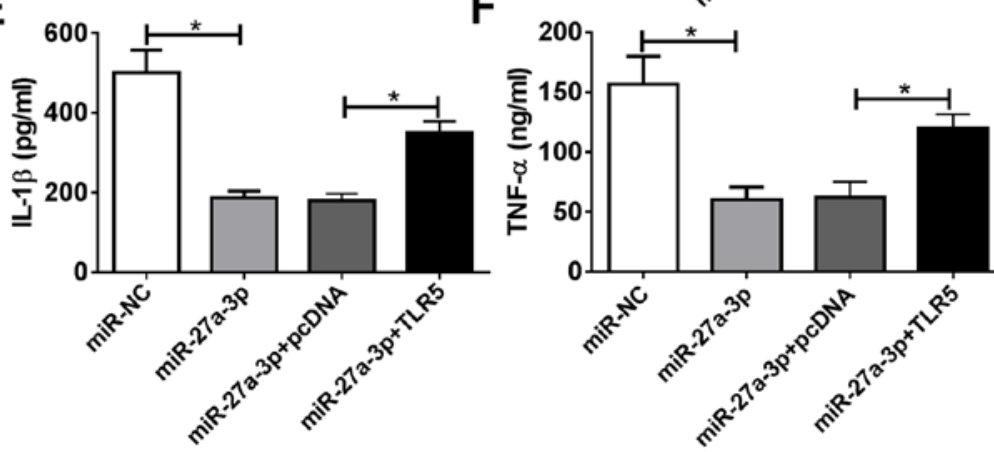

Figure 5. Influence of TLR5 overexpression on miR-27a-3p-induced effects in RASFs. RASFs were co-transfected with miR-27a-3p and pcDNA-TLR5 (TLR5) vector or pcDNA vector. (A) Western blotting detected the TLR5 level. (B and C) Cell proliferation and apoptosis were investigated following transfection using MTT assay and flow cytometry. (D-F) Products of IL-6, IL-1 $\beta$ and TNF- $\alpha$ were determined by ELISA kits. "P<0.05, compared with miR-NC or miR-27a-3p+pcDNA. TLR5, toll-like receptor 5; miR, microRNA; RASFs, RA synovial fibroblasts; IL, interleukin; TNF, tumor necrosis factor; NC, negative control.

endothelial growth factor, matrix metalloproteinase (MMP)-3 and MMP-9 (30).

However, loss of balance in RASF proliferation and apoptosis also served an essential role in RA pathogenesis.
Furthermore, the activity of RASFs conduced to inflammatory response, thereby driving the progression of RA into late stages $(31,32)$. For example, miR-140-5p prevented the proliferation and secretion of IL-6 and IL-8 in RASFs by targeting 


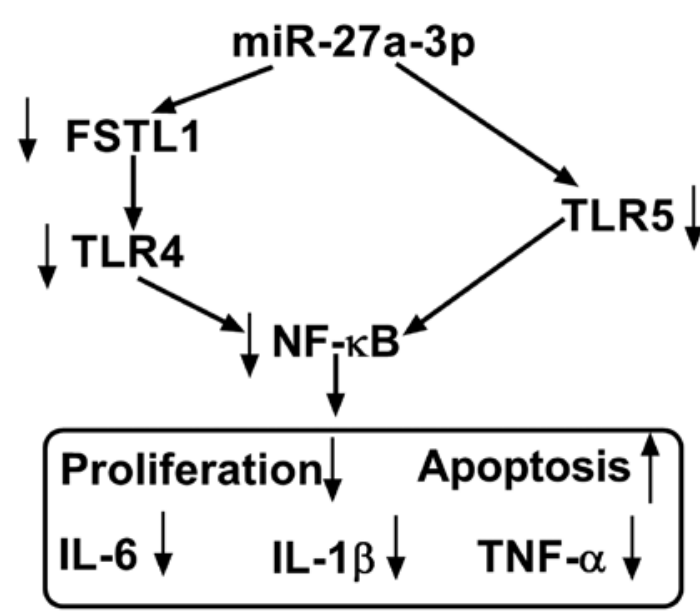

Figure 6. The molecular function and mechanism of miR-27a-3p in human RASFs. Upregulating miR-27a-3p inhibited TLR5 expression to attenuate proliferation and pro-inflammatory cytokines secretion, as well as to promote apoptosis. miR, microRNA; RASFs, RA synovial fibroblasts; TLR5, toll-like receptor 5; IL, interleukin; TNF, tumor necrosis factor.

TLR4 (33). Fu et al (34) demonstrated the suppressive role of miR-3926 in RASF proliferation and pro-inflammatory factors (IL-1 $\beta$, IL- 6 and TNF- $\alpha$ ) secretion through targeting TLR5. The present study focused on the contribution of miR-27a-3p to RASF proliferation, apoptosis and inflammation, instead of the migration and invasion (21). As a result, re-expressing miR-27a-3p may increase the apoptotic rate and attenuate the proliferation ability and secretions of IL-1 $\beta$, IL- 6 and TNF- $\alpha$ in human RASFs ex-vivo (Fig. 6). Furthermore, inhibiting TLR5 displayed a suppressive role in RASF proliferation and inflammation, and restoring TLR5 mitigated the anti-proliferation and anti-inflammation role of miR-27a-3p-overexpression in RASFs. Notably, TLR5 was identified as a novel target gene for miR-27a-3p. Furthermore, Shi et al (21) demonstrated that miR-27a-3p inhibited cell migration and invasion of RASFs by

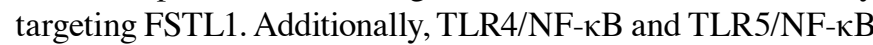
signaling pathways had been discovered in fibroblasts $(21,35)$. Therefore, we hypothesized a possible miR-27a-3p/TLRs/NF-kB regulatory mechanism underlying RASFs proliferation, apoptosis, inflammation, migration and invasion (Fig. 6). According to aforementioned results, miRNA expression alteration, including restoring miR-27a-3p, was considered to be a potential therapeutic strategy for RA treatment.

TLR5 is a crucial member of the TLR family, which was evolutionarily conserved innate immune receptors (36). TLRs serves a fundamental role in the first-line defense against foreign molecules, and TLRs were released following injury or pathogen infection. The functions of TLR $1 / 2 / 4 / 5$ and 7 had been extensively studied in RA $(37,38)$. However, function of TLR5 in RA remain unknown. TLR5 was known to specifically sense and recognize flagellin, the major structural protein of bacterial flagella (39). For example, TLR5 was implicated in the pathogenesis of autoimmune diseases, including systemic lupus erythematosus and RA $(37,40)$. Chamberlain et al (41) demonstrated that TLR5 expression in myeloid was positively associated with TNF level and RA disease activity (DAS28). Kim et al (42) reported that ligation of TLR 5 may promote myeloid cell infiltration and differentiation into mature osteoclasts in RA and experimental arthritis. Furthermore, Fu et al (34) demonstrated that the miR-3926/TLR5 pathway may represent a novel target for the prevention and treatment of RA. The present study observed a high expression of TLR5 in human RA synovial tissues and RASFs, and this data supported the results of a previous study (34). Additionally, low expression of TLR5 not only suppressed proliferation and secretions of TNF- $\alpha$, IL-1 $\beta$ and IL- 6 , but also promoted the apoptotic rate of RASFs ex-vivo, which were in consistent with the results of a previous study (34). Furthermore, it was identified that TLR5 was a downstream target of miR-27a-3p.

In conclusion, the present study highlighted that overexpressing miR-27a-3p and/or silencing TLR5 may interfere with RA progression by inhibiting RASF proliferation and inflammatory response and facilitating apoptosis (Fig. 6). The results of the present study may provide a potential therapeutic target for RA. Meanwhile, a novel miR-27a-3p/TLR5 axis was discovered underlying RASF dysfunction; however, the precise signaling pathway of miR-27a-3p/TLR5, including $\mathrm{NF}-\mathrm{kB}$, should be better investigated in the future.

\section{Acknowledgements}

Not applicable.

\section{Funding}

No funding was received.

\section{Availability of data and materials}

The datasets used and/or analyzed during the present study are available from the corresponding author on reasonable request.

\section{Authors' contributions}

LC and QL conceived the study, designed the methodology and performed the final analysis. QL, JC, RF and CY performed the experiments and acquired the data. QL and JC confirmed the authenticity of all the raw data and performed statistical analysis. LC and QL wrote the original draft. LC reviewed and edited the manuscript. All authors read and approved the final manuscript.

\section{Ethics approval and consent to participate}

The present study was conducted in accordance with the Declaration of Helsinki and was approved by the Research Ethics Committee of General Hospital of Central Theater Command (Wuhan, China). Written informed consent was obtained from each patient.

\section{Patient consent for participation}

Not applicable.

\section{Competing interests}

The authors declare that they have no competing interests. 


\section{References}

1. Coras R, Narasimhan R and Guma M: Liquid biopsies to guide therapeutic decisions in rheumatoid arthritis. Transl Res 201: 1-12, 2018.

2. Araki Y and Mimura T: The mechanisms underlying chronic inflammation in rheumatoid arthritis from the perspective of the epigenetic landscape. J Immunol Res 2016: 6290682, 2016.

3. Croia C, Bursi R, Sutera D, Petrelli F, Alunno A and Puxeddu I: One year in review 2019: Pathogenesis of rheumatoid arthritis. Clin Exp Rheumatol 37: 347-357, 2019.

4. Fang Q, Zhou C and Nandakumar KS: Molecular and cellular pathways contributing to joint damage in rheumatoid arthritis Mediators Inflamm 2020: 3830212, 2020.

5. Alunno A, Carubbi F, Giacomelli R and Gerli R: Cytokines in the pathogenesis of rheumatoid arthritis: New players and therapeutic targets. BMC Rheumatol 1: 3, 2017.

6. Falconer J, Murphy AN, Young SP, Clark AR, Tiziani S, Guma M and Buckley CD: Review: Synovial cell metabolism and chronic inflammation in rheumatoid arthritis. Arthritis Rheumatol 70 : 984-999, 2018.

7. Shi J,Ermann J, Weissman BN, Smith SEand Mandell JC: Thinking beyond pannus: A review of retro-odontoid pseudotumor due to rheumatoid and non-rheumatoid etiologies. Skeletal Radiol 48 $1511-1523,2019$

8. Laev SS and Salakhutdinov NF: Anti-arthritic agents: Progress and potential. Bioorg Med Chem 23: 3059-3080, 2015.

9. Yuasa S, Yamaguchi H, Nakanishi Y, Kawaminami S, Tabata R, Shimizu N, Kohno M, Shimizu T, Miyata J, Nakayama M, et al: Treatment responses and their predictors in patients with rheumatoid arthritis treated with biological agents. J Med Invest 60: 77-90, 2013.

10. Zamanpoor M: The genetic pathogenesis, diagnosis and therapeutic insight of rheumatoid arthritis. Clin Genet 95: 547-557, 2019

11. Moran-Moguel MC, Petarra-Del Rio S, Mayorquin-Galvan EE and Zavala-Cerna MG: Rheumatoid arthritis and mirnas: A critical review through a functional view. J Immunol Res 2018 : 2474529, 2018

12. Dong H, Lei J, Ding L, Wen Y, Ju H and Zhang X: MicroRNA: Function, detection, and bioanalysis. Chem Rev 113: 6207-6233, 2013.

13. Dudics S, Venkatesha SH and Moudgil KD: The micro-RNA expression profiles of autoimmune arthritis reveal novel biomarkers of the disease and therapeutic response. Int J Mol Sci 19: 19, 2018

14. Zhang H, Huang X, Ye L, Guo G, Li X, Chen C, Sun L, Li B, Chen $\mathrm{N}$ and Xue X: B cell-related circulating MicroRNAs with the potential value of biomarkers in the differential diagnosis, and distinguishment between the disease activity and lupus nephritis for systemic lupus erythematosus. Front Immunol 9 : 1473, 2018.

15. Regev K, Paul A, Healy B, von Glenn F, Diaz-Cruz C, Gholipour T, Mazzola MA, Raheja R, Nejad P, Glanz BI, et al: Comprehensive evaluation of serum microRNAs as biomarkers in multiple sclerosis. Neurol Neuroimmunol Neuroinflamm 3: e267, 2016.

16. Evangelatos G, Fragoulis GE, Koulouri V and Lambrou GI: MicroRNAs in rheumatoid arthritis: From pathogenesis to clinical impact. Autoimmun Rev 18: 102391, 2019.

17. Sharma AR, Sharma G, Lee SS and Chakraborty C: miRNAregulated key components of cytokine signaling pathways and inflammation in rheumatoid arthritis. Med Res Rev 36: 425-439, 2016.

18. Sode J, Krintel SB, Carlsen AL, Hetland ML, Johansen JS, Hørslev-Petersen K, Stengaard-Pedersen K, Ellingsen T, Burton M, Junker P, et al: Plasma MicroRNA profiles in patients with early rheumatoid arthritis responding to adalimumab plus methotrexate vs, methotrexate alone: A Placebo-controlled Clinical Trial. J Rheumatol 45: 53-61, 2018.

19. Takamura Y, Aoki W, Satomura A, Shibasaki S and Ueda M Small RNAs detected in exosomes derived from the MH7A synovial fibroblast cell line with TNF- $\alpha$ stimulation. PLoS One 13 e0201851, 2018.

20. Maeda Y, Farina NH, Matzelle MM, Fanning PJ, Lian JB, Gravallese EM: Synovium-derived MicroRNAs regulate bone pathways in rheumatoid arthritis. J Bone Miner Res 32: 461-472, 2017.

21. Shi DL, Shi GR, Xie J, Du XZ and Yang H: MicroRNA-27a inhibits cell migration and invasion of fibroblast-like synoviocytes by targeting follistatin-like protein 1 in rheumatoid arthritis. Mol Cells 39: 611-618, 2016.
22. Aletaha D, Neogi T, Silman AJ, Funovits J, Felson DT, Bingham CO III, Birnbaum NS, Burmester GR, Bykerk VP, Cohen MD, et al: 2010 rheumatoid arthritis classification criteria: An American College of Rheumatology/European League Against Rheumatism collaborative initiative. Ann Rheum Dis 69: 1580-1588, 2010.

23. Li XJ, Xu M, Zhao XQ, Zhao JN, Chen FF, Yu W, Gao DY and Luo B: Proteomic analysis of synovial fibroblast-like synoviocytes from rheumatoid arthritis. Clin Exp Rheumatol 31: 552-558, 2013.

24. Livak KJ and Schmittgen TD: Analysis of relative gene expression data using real-time quantitative PCR and the 2(-Delta Delta C(T)) method. Methods 25: 402-408, 2001.

25. Zou Y, Xu S, Xiao Y, Qiu Q, Shi M, Wang J, Liang L, Zhan Z, Yang $\mathrm{X}$, Olsen N, et al: Long noncoding RNA LERFS negatively regulates rheumatoid synovial aggression and proliferation. J Clin Invest 128: 4510-4524, 2018.

26. Alamanos Y and Drosos AA: Epidemiology of adult rheumatoid arthritis. Autoimmun Rev 4: 130-136, 2005.

27. Liu Y, Pan YF, Xue YQ, Fang LK, Guo XH, Guo X, Liu M, Mo BY, Yang MR, Liu F, et al: uPAR promotes tumor-like biologic behaviors of fibroblast-like synoviocytes through PI3K/Akt signaling pathway in patients with rheumatoid arthritis. Cell Mol Immunol 15: 171-181, 2018

28. Cai P, Jiang T, Li B, Qin X,Lu Z, Le Y, Shen C, Yang Y, Zheng L and Zhao J: Comparison of rheumatoid arthritis (RA) and osteoarthritis (OA) based on microarray profiles of human joint fibroblast-like synoviocytes. Cell Biochem Funct 37: 31-41, 2019.

29. Ye Y, Gao X and Yang N: LncRNA ZFAS1 promotes cell migration and invasion of fibroblast-like synoviocytes by suppression of miR-27a in rheumatoid arthritis. Hum Cell 31: 14-21, 2018.

30. Yang S and Yang Y: Downregulation of microRNA 221 decreases migration and invasion in fibroblast like synoviocytes in rheumatoid arthritis. Mol Med Rep 12: 2395-2401, 2015.

31. Neumann E,Lefèvre S,Zimmermann B, Gay S and Müller-Ladner U: Rheumatoid arthritis progression mediated by activated synovial fibroblasts. Trends Mol Med 16: 458-468, 2010.

32. Karouzakis E, Gay RE, Gay S and Neidhart M: Epigenetic control in rheumatoid arthritis synovial fibroblasts. Nat Rev Rheumatol 5: 266-272, 2009.

33. Li H, Guan SB, Lu Y and Wang F: MiR-140-5p inhibits synovial fibroblasts proliferation and inflammatory cytokines secretion through targeting TLR4. Biomed Pharmacother 96: 208-214, 2017.

34. Fu D, Xiao C, Xie Y, Gao J and Ye S: MiR-3926 inhibits synovial fibroblasts proliferation and inflammatory cytokines secretion through targeting toll like receptor 5. Gene 687: 200-206, 2019.

35. Thunyakitpisal P, Ruangpornvisuti V, Kengkwasing P, Chokboribal J and Sangvanich P: Acemannan increases NF- $\kappa \mathrm{B} / \mathrm{DNA}$ binding and IL-6/-8 expression by selectively binding Toll-like receptor-5 in human gingival fibroblasts. Carbohydr Polym 161: 149-157, 2017.

36. Sabroe I, Read RC, Whyte MK, Dockrell DH, Vogel SN and Dower SK: Toll-like receptors in health and disease: Complex questions remain. J Immunol 171: 1630-1635, 2003.

37. Elshabrawy HA, Essani AE, Szekanecz Z, Fox DA and Shahrara S: TLRs, future potential therapeutic targets for RA. Autoimmun Rev 16: 103-113, 2017.

38. Thwaites RS, Unterberger S, Chamberlain G, Walker-Bone K, Davies KA and Sacre S: TLR1/2 and 5 induce elevated cytokine levels from rheumatoid arthritis monocytes independent of ACPA or RF autoantibody status. Rheumatology (Oxford) 59: 3533-3539, 2020.

39. Smith KD, Andersen-Nissen E, Hayashi F, Strobe K, Bergman MA, Barrett SL, Cookson BT and Aderem A: Toll-like receptor 5 recognizes a conserved site on flagellin required for protofilament formation and bacterial motility. Nat Immunol 4: 1247-1253, 2003.

40. Wu YW, Tang W and Zuo JP: Toll-like receptors: Potential targets for lupus treatment. Acta Pharmacol Sin 36: 1395-1407, 2015.

41. Chamberlain ND, Vila OM, Volin MV, Volin MV, Volkov S, Pope RM, Swedler W, Mandelin AM 2nd, Shahrara S, et al: TLR5, a novel and unidentified inflammatory mediator in rheumatoid arthritis that correlates with disease activity score and joint TNF- $\alpha$ levels. J Immunol 189: 475-483, 2012.

42. Kim SJ, Chen Z, Chamberlain ND, Essani AB, Volin MV, Amin MA, Volkov S, Gravallese EM, Arami S, Swedler W, et al: Ligation of TLR5 promotes myeloid cell infiltration and differentiation into mature osteoclasts in rheumatoid arthritis and experimental arthritis. J Immunol 193: 3902-3913, 2014.

This work is licensed under a Creative Commons Attribution-NonCommercial-NoDerivatives 4.0 International (CC BY-NC-ND 4.0) License. 